\title{
Efficacy of a 6-Week Supervised Strengthening Exercise Program with EMG-Biofeedback in Patients with Muscular Dystrophy: a Randomized Controlled Trial
}

\author{
N. Maghbouli ${ }^{1}$, N. Shirzad ${ }^{2}$, H. R. Fateh ${ }^{3}$, F. Fatehi ${ }^{4}$, S. Z. Emami Razavi ${ }^{5}$, S. Nafissi ${ }^{6}$ \\ 1 Department of Physical Medicine and Rehabilitation, School of Medicine, Tehran University of Medical Sciences, \\ Tehran, Iran \\ 2 Department of Occupational Therapy, School of Rehabilitation, Shahid Beheshti University of Medical Sciences, \\ Tehran, Iran \\ 3 Department of Physical Medicine and Rehabilitation, Shariati Hospital, Tehran University of Medical Sciences, \\ Tehran, Iran \\ ${ }^{4}$ Department of Neurology, Iranian Center of Neurological Research, Neuroscience Institute, Shariati Hospital, \\ Tehran University of Medical Sciences, Tehran, Iran \\ ${ }^{5}$ Department of Physical Medicine and Rehabilitation, Imam Khomeini Hospital, Tehran University of Medical \\ Sciences, Tehran, Iran \\ ${ }^{6}$ Department of Neurology, Shariati Hospital, Tehran University of Medical Sciences, Tehran, Iran
}

\section{CORRESPONDING AUTHOR:}

\section{Hamid R. Fateh}

Department of Physical Medicine and

Rehabilitation

Shariati Hospital

Tehran University of Medical Sciences

Kargar Avenue

14117 Tehran, Iran

E-mail: hr-fateh@tums.ac.ir

\section{DOI:}

10.32098/mltj.04.2021.16

LEVEL OF EVIDENCE: 1B

\section{ABBREVIATIONS:}

MD: Muscular Dystrophy

EMG-BFB: Electromyographic Biofeedback

CG: Control Group

BBS: Berg Balance Scale

MFM-32: Motor Function Measure-32

FSS: Fatigue Severity Scale

TUG: Timed Up Go test

SCT: Stair Climb Test

SST: Stand up from Supine position Test

\section{SUMMARY}

Background. Although previous trials investigated the effectiveness of exercise training programs in muscular dystrophy (MD) patients, we faced a lack of evidence on biofeedback. This study aimed to assess the safety and efficacy of a 6-week supervised strengthening exercise training program with electromyography-Biofeedback (EMG-BFB) on muscle strength, motor function, and balance of MD patients.

Methods. Forty MD patients were assigned randomly to EMG-BFB group $(n=20)$ and supervised strengthening exercise program as the control group (CG). Training strengthening program consisted of 12 sessions twice weekly for 30 minutes for 6 weeks. Patients in EMG-BFB group did exercises using biofeedback. Patients in CG also performed conventional exercises under the supervision of therapist for 6 weeks. The primary outcome was change in muscle strength. Other secondary outcomes were fatigue severity test, Vignos scale, timed up go test, stair climb test, stand up from supine position test, Berg balance scale, and quality of life. All outcomes measured pre- and post-intervention and 3 months after program.

Results. The compliance rate was $60 \%$ in this study. Significant improvements with training were observed in the hip muscles strength and Berg balance scale (BBS), motor function measure-32, and fatigue scale by week 6 and further to week 12 in both groups. Only the BBS improvement showed significant change in the intervention group in comparison to the control group $(\mathrm{P}=0.040)$, which was not correlated with muscle strengths $(\mathrm{P}=0.420)$.

Conclusions. This study demonstrated benefits of a supervised strengthening exercise program in MD patients. These benefits seem to be occurred without any adverse effects to cause patients to drop out of the study. According to some balance improvements, EMG-Biofeedback might be helpful in rehabilitation programs of MD patients.

\section{KEY WORDS}

Muscular dystrophy; function; strength; balance; rehabilitation; biofeedback. 


\section{INTRODUCTION}

Muscular dystrophies (MDs) are a group of heterogeneous inherited diseases affecting cytoskeletal proteins causing progressive weakness (1). Muscle atrophy and dysfunction lead to significant functional impairments, including motor functions and swallowing difficulties, deformities such as scoliosis, respiratory alterations, and cardiac events (1). Their reduced physical activity contributes to deconditioning and increased fatigue perception that causes mental problems and social isolation (2). Falling is a frequent event among MD patients with catastrophic consequences like musculoskeletal injuries, fractures, and falling recurrence phobia, which aggregate their functional status (3).

Literature showed a growing body of evidence in support of exercise role in neuromuscular diseases incredibly muscular dystrophies (4). Although in ancient studies, doubts were raised about the possibility of muscle fiber damage by exercise among muscular dystrophy patients, recent studies using objective measures including serum Creatine-kinase level, Syndecans expression, ultrasonographic findings and T2-MRI muscle signal change following exercise proved its safety for this group of patients (5-8). During the literature review, we identified seven controlled trials investigating exercise effectiveness on MDs, three evaluating aerobic exercises (9-11), three strength-training (12-14), and one consisted of both exercise type (15) focusing mainly on FSHD and myotonic dystrophy subtypes. Aerobic exercises have been proved to be applicable on aerobic capacity and quality of life among MD patients (9-11). Controversies existed on strengthening exercises; however, consensus on resistive exercises with mild intensity was founded (12-14). One trial suggested combined aerobic and strengthening exercise as a more effective method than aerobic training alone (15). Most studies in this regard are with inappropriate methodology, insufficient sample size, with short-time follow-up.

The original biofeedback focusing on static muscle training and movement changed its place to novel biofeedback systems with advanced cue methods and control systems for task-oriented biofeedback (16). Although biofeedback is introduced to improve motor control through functional training among neuro-rehabilitation patients (17), its usefulness in motor control is reasonable in neuromuscular patients. Limited studies existed on the effects of biofeedback on neuromuscular diseases, and to our knowledge, there is no study on its effectiveness on MDs which approves novelty of this study.

Therefore, a randomized controlled trial was conducted to investigate the efficacy of 6-week strengthening exercises using electromyographic biofeedback (EMG-BFB) on muscular dystrophy patients evaluating muscle strength, motor function, and balance.

\section{METHODS}

\section{Study design}

This study is a randomized clinical trial with a two-arm and allocation ratio of $1: 1$, implemented in the physical medicine and rehabilitation $(P M \& R)$ clinic of Shariati hospital in Tehran University of Medical Sciences (TUMS) from May 2020 to February 2021. The protocol of this study is approved by the ethics committee of TUMS (code: IR.TUMS.MEDICINE.REC.1398.031) and registered in the Iranian Registry of Clinical Trials Database (IRCT20200326046864N1). This study meets the ethical standards of the Muscles, Ligaments and Tendons Journal (18). After trial commencement, low compliance of patients for follow-up has been supposed due to COVID-19 pandemic, therefore considering patient retention, we prepared some medical consultation about COVID-19 for participants.

\section{Sample size calculation}

The primary outcome measure of this study was muscle strength; so, the sample size was calculated with a statistical level of significance; 0.05 , and test power of 0.8 using repeated-measures ANOVA. In conclusion, 12 participants were calculated for each group. Considering the high probability of patients numbers falling during the intervention and follow-up process, we entered 20 patients for each group.

\section{Inclusion criteria}

We recruited all subjects with $\geq 18$ years of age and proximal muscles involvement with at least 10 meter walking ability without assist whose diagnosis were confirmed by a neuromuscular fellowship and genetic tests. Ethically each patient signed the informed consent form before participation in the study.

\section{Exclusion criteria}

Our exclusion criteria were recent history of trauma or surgery or acute event during the past three months, history of other neurologic or systematic disorders, use of drugs affecting muscles within three months. Participants with auditory or vision problems, cognition impairment, and cardiomyopathy were excluded. Patients were asked to inform the therapist if they tended to participate in other rehabilitation programs during the study. 


\section{Intervention}

Patients were randomly assigned to two groups; the conventional strengthening exercise and EMG-BFB groups. Simple randomization, using a random number table, was applied as a randomized assignment method. The researcher (NM) selected the randomized number and assigned it to the specific group.

Before all interventions, demographic information such as age, gender, height, weight, and drug history was gathered. In the first session, muscle strength and functional tests were recorded, and further intervention sessions were set. The intervention group received strengthening exercises using EMG-BFB (Saebo MyoTrac Infiniti, threshold setting: $50 \%$ of maximum contraction) for 6 weeks, twice weekly, for 30 minutes with at least 48 hours interval between training sessions. The control group with the same schedule performed the exercises under a therapist supervision. Isometric strengthening exercises involved hip flexors, extensors, adductors, and abductors in addition to knee flexors and extensors, and EMG-BFB intensity was set for $50 \%$ of maximum voluntary contraction. Furthermore, they were also educated on lifestyle modifications and strengthening exercises, verbal and through a brochure, filling checklist, and evaluating exercise adherence for more 6 weeks after intervention until the last assessment. Muscle strength and functional tests were assessed after the 12 -session intervention for 6 weeks for the second time and 3 months after the beginning of the program for the third time. Previous studies suggested low to moderate intensity of exercises for MD patients (14). We used the Borg scale for intensity evaluation during the intervention, and the program was stopped if Borg $>14$. Muscular cramp or pain during or after exercise were other indications of program discontinuation.

\section{Outcome measurements}

The primary outcome measure was muscle strength which was measured by one examiner (N.S) in all patients, using a handheld dynamometer type CT 3001 (CITEC handheld dynamometer, C.I.T. Technics, Groningen, The Netherlands) (19). This tool is more sensitive than manual testing for moderate impairments (manually quoted at 4 and higher). By using verbal encouragement, forces are recorded 3 times for each muscle group, and the mean measure was used for analysis. Intra-rater variability of strength testing was below $10 \%$.

Secondary outcome measures were the motor function measure-32 (MFM-32), fatigue severity scale (FSS), Vignos scale, timed up go test (TUG), stair climb test (SCT), stand up from supine position test (SST) and Berg balance scale
(BBS). MFM-32 is a scale for evaluation of motor function of neuromuscular patients consist 32 items with 4-point Likert scoring for each item (20). FSS is used for assessment of fatigue perception and its effects on life and functions which includes 9 items with 7-point for each item (21). Lower limbs function evaluated by Vignos scale. It scores 1 to 10 in which score 1 means ability to walk and climbing up without assistance and score 10 means laying on the bed (22). Time needed for walking 10 meters in moderate speed (23) and going 3 steps up and down (24) assessed via TUG and SCT, respectively. SST specifies the time needed to stand from supine position and sit (25). BBS as a known scale used for balance evaluation and it consists 14 items with 4-point Likert scoring for each item (26). To survey quality of life, SF-36 questionnaire was used. This tool consists 36 items and scores between 0-100 including 8 subscales: physical function, physical role, bodily pain, general health, social function, emotional role, mental health, and vitality (23).

\section{Data analysis}

Statistical Analyses were performed by Stata software version 16. A P-value of less than 0.05 was considered significant. All analyses were performed on 12 patients per group. Categorical data are presented as percent, and Continuous demographic variables are presented as Mean \pm Standard deviation or Median (Interquartile range). For Further analyses, Shapiro-Wilk and Levene's tests, respectively, were used for normal data distribution and variance homogeneity assessment. Two-way repeated-measures ANOVAs were performed to analyze changes between IG and CG at each time point. Bonferroni multiple comparison procedure was used for post-hoc analysis. Pearson's correlation coefficient was performed to determine relationships between outcomes.

\section{RESULTS}

A total of 76 patients are assessed from the point of inclusion criteria, and they were called for participation in this study, and 40 persons were accepted and randomized into two groups. Eleven patients left the study during training sessions for reasons such as transportation problems, COVID-19 related quarantine, being affected by COVID19, or fear of COVID-19 infection. Five patients did not come back for the final assessment, which was after 3 months from the rehabilitation program. Finally, data of 24 patients were analyzed who were completed all training and assessment sessions (figure 1). Four patients had Becker muscular dystrophy, 8 persons facioscapulohumeral dystrophy, 10 participants limb-girdle muscular dystrophy, and 2 persons 


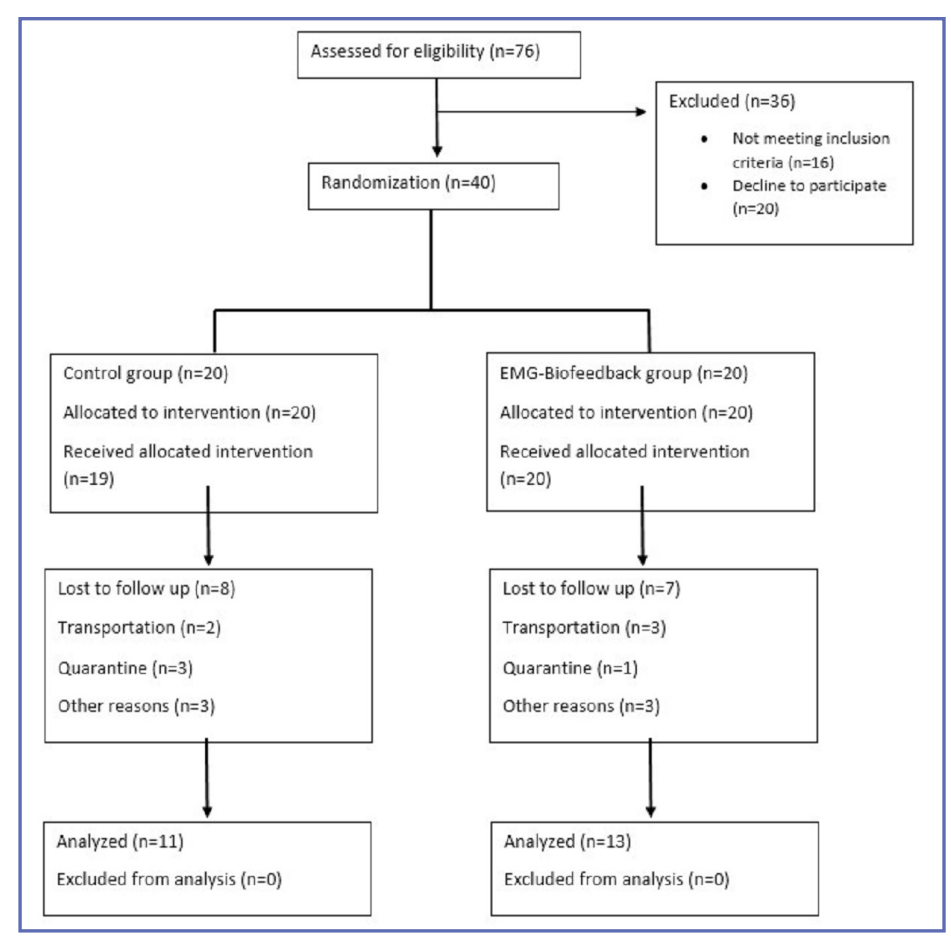

Figure 1. Trial chart. myotonic dystrophy. Approximately 25\% of participants were women, and the mean of participants' age was $35.1 \pm 5.4$. Table I shows demographic data, initial muscle strength and functional tests of participants, and comparison between two groups. There were no statistically significant differences between the two groups, and participants' characteristics were similar in both groups. No complication was reported during or after training sessions, and there was no need for exercise discontinuation for none of the participants.

Figure 2 shows changing pattern of functional tests before, after, and 3 months after the intervention. The MFM-32, FSS and BBS were improved significantly in both CG and EMG-BFB group during all 3 assessments. The SST, SCT and TUG tests showed some improvements in both groups after the intervention, but this improvement was not observed in the last evaluation after 3 months. The Vignos test was not changed significantly after training sessions in both groups. Figure 3 reports results of functional tests comparing between two groups, CG and EMG-BFB. Based on figure, SST and BBS showed better results in the intervention group, although other tests resulted in

Table I. Characteristics of participants.

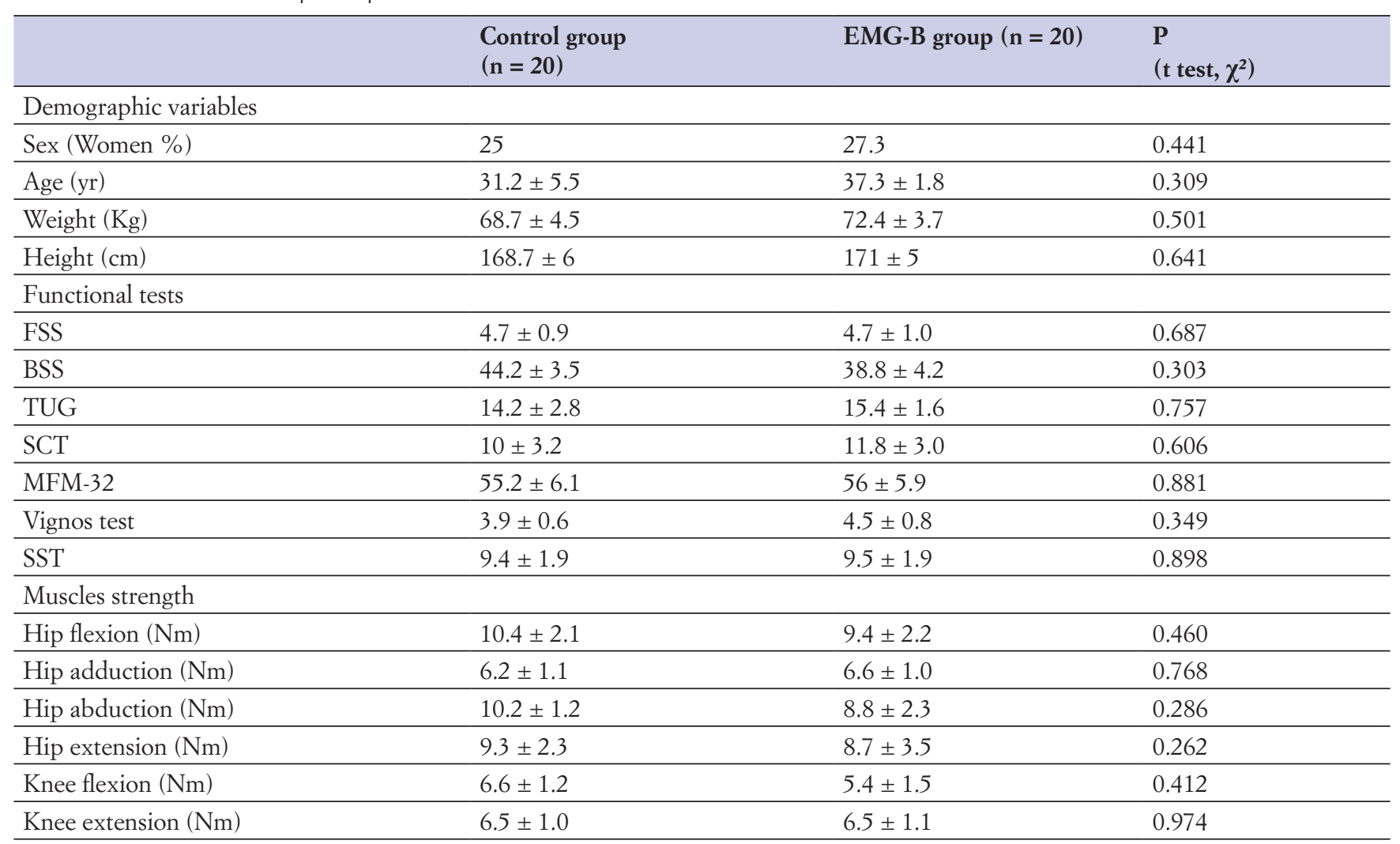




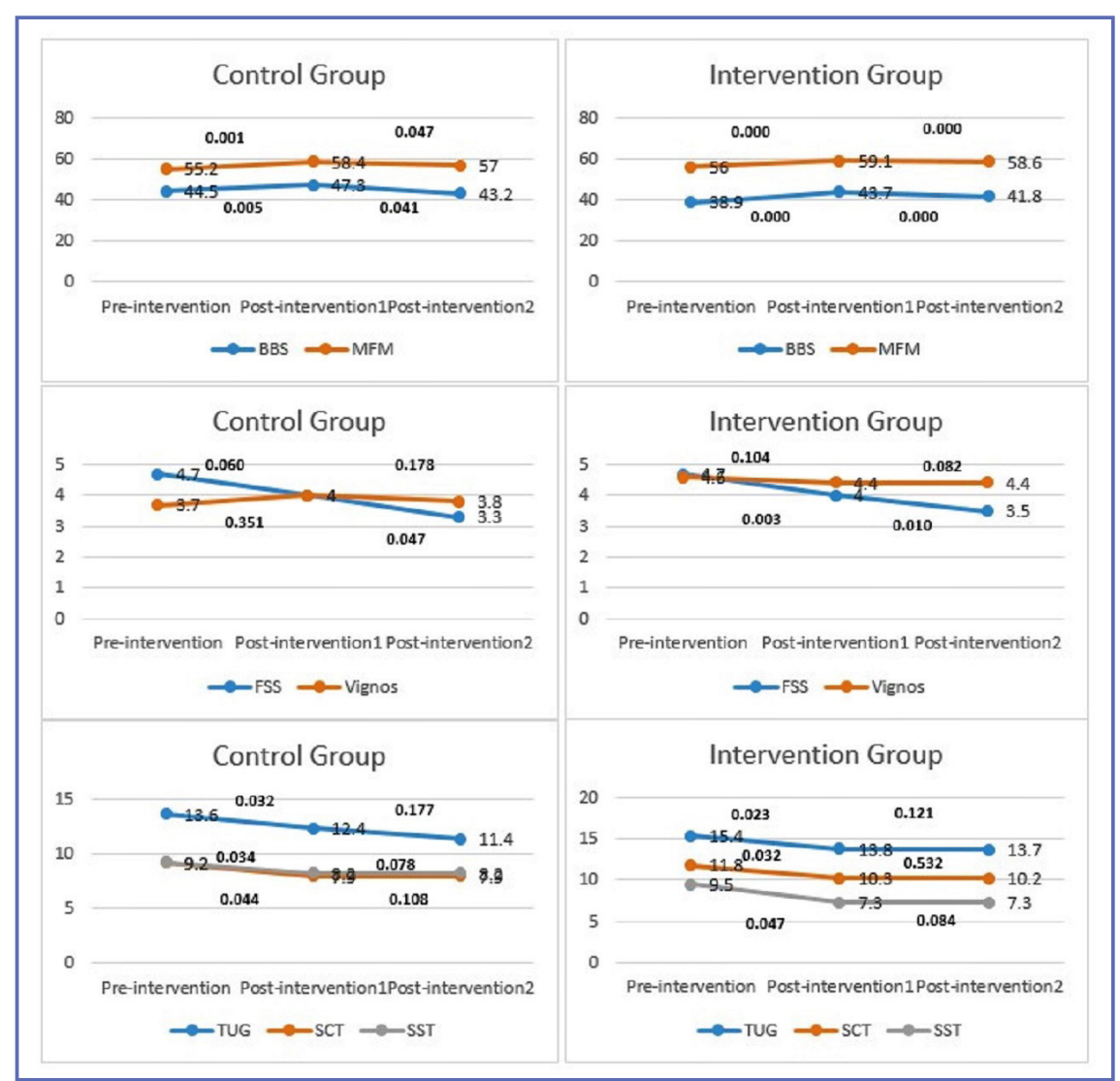

Figure 2. MFM-32, BBS, Vignos test, FSS, TUG, SCT and SST changes during three assessments in both EMG-BFB group and CG.

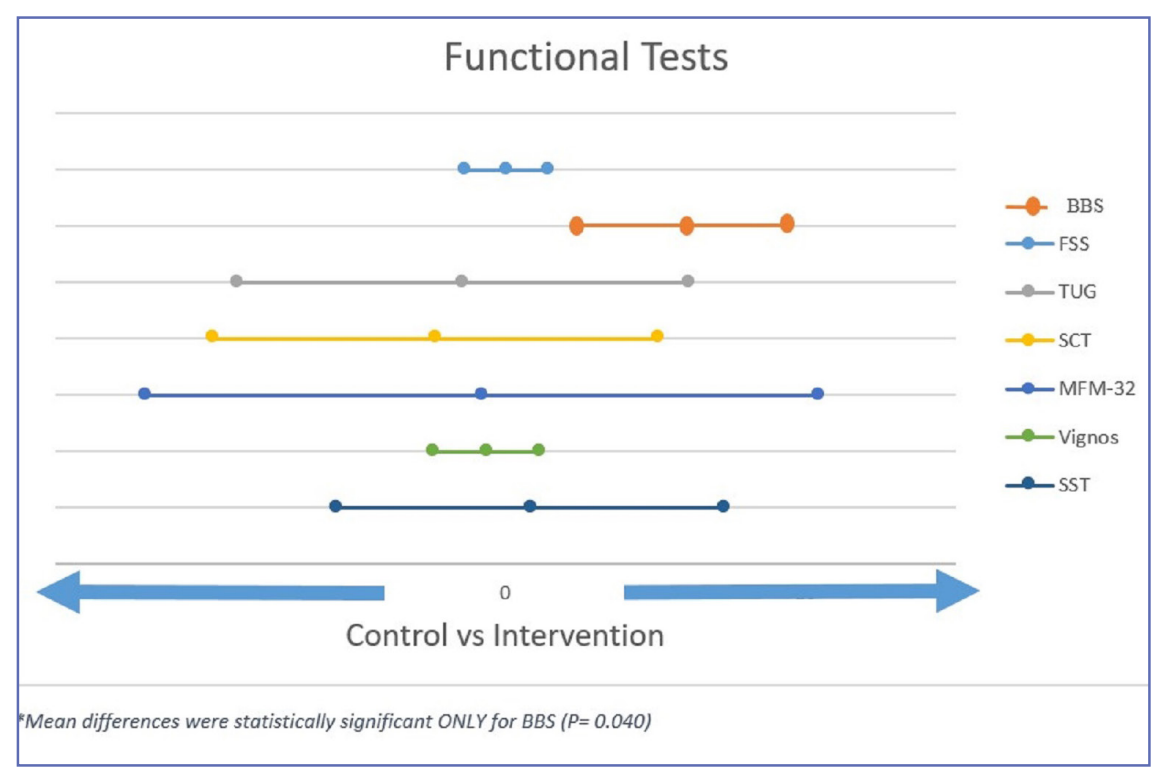

Figure 3. Functional tests mean differences between intervention vs control groups. better findings in CG. Finally, only BBS showed statistically significant improvement toward the EMG-BFB $(\mathrm{P}=0.040)$. While evaluating relationship between BBS and muscle strength and functional tests, significant association was found between BBS and TUG $(\mathrm{r}=-0.733, \mathrm{p}$ $=0.000)$, BBS and MFM-32 $(r=0.637$, $\mathrm{p}=0.001)$, BBS and SST $(\mathrm{r}=-0.441, \mathrm{p}$ $=0.021$ ), but significant relationship was not detected between BBS and muscle strengths $(\mathrm{P}=0.420)$.

Table II shows muscle strength changes during three assessments in both groups. In CG and EMG-BFB group, hip flexion, abduction, adduction, and extension strengths were improved after the intervention. Significant differences were not detected between the two groups comparing the EMG-BFB group and CG.

Table III shows the SF-36 results of participants. Considering table data, physical function, bodily pain, social function, emotional role, and mental health subscales improved after intervention in the EMG-BFB group, but no improvement is seen in CG. In the EMG-BFB group, physical and general health showed better results than CG $(\mathrm{P}=0.034,0.037)$.

Subgroup analysis based on dystrophy type suggested no differences between different types, neither in muscle strength improvements nor functional tests.

\section{DISCUSSION}

To the our knowledge, this study evaluated, for the first time, the safety and efficacy of strengthening exercises using EMG-biofeedback for MD patients through a randomized clinical trial on muscle strength, motor function, balance, and quality of life over 12 weeks. The main findings of this study included muscle strength, motor function, balance, and some aspects of quality of life improvement following intervention and decreased experienced fatigue. 
Table II. Muscle strength change before and after intervention in EMG-BFB and CG.

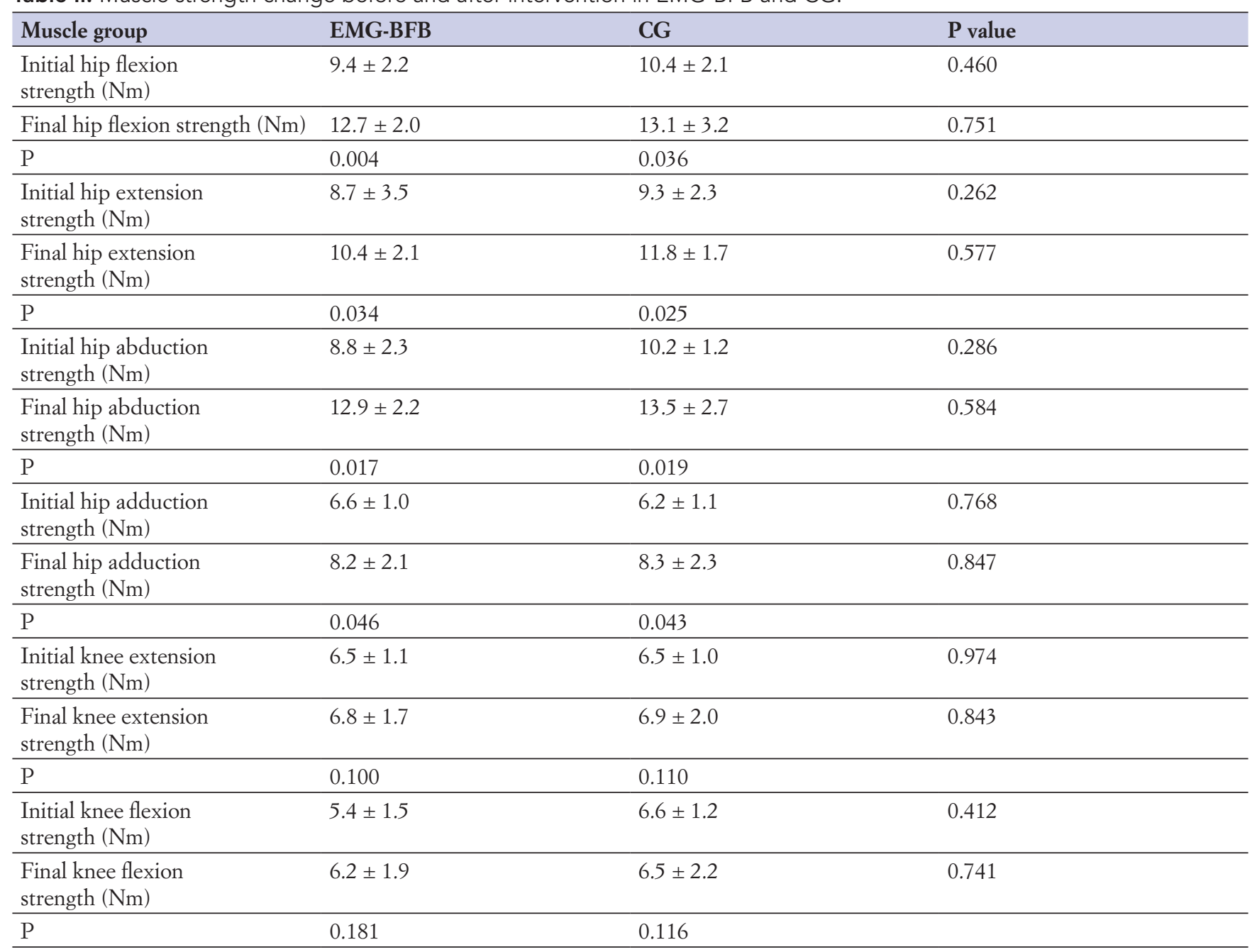

Table III. Results of quality of life questionnaire and its subscales.

\begin{tabular}{lllllll}
\hline Subscale & $\begin{array}{l}\text { Intergroup } \\
\text { difference } \\
\text { for Control }\end{array}$ & P value & $\begin{array}{l}\text { Intergroup } \\
\text { difference for } \\
\text { Intervention }\end{array}$ & P value & $\begin{array}{l}\text { Between groups } \\
\text { differences }\end{array}$ & P value \\
\hline Physical Function & $6.4(-3.5,16.3)$ & 0.163 & $10(4.6,15.3)$ & 0.001 & $3.2(-10.6,17.1)$ & 0.634 \\
\hline Role of Physical & $2.1(-0.3,4.6)$ & 0.078 & $1.7(-3.5,5.8)$ & 0.355 & $13.5(1.1,26.1)$ & 0.034 \\
\hline Bodily Pain & $4.3(-0.1,8.4)$ & 0.055 & $10.7(5.7,15.6)$ & 0.000 & $2.1(-8.4,12.7)$ & 0.678 \\
\hline General Health & $1.8(-2,5.7)$ & 0.284 & $1.6(-5.4,13.4)$ & 0.133 & $10.4(1.7,20.2)$ & 0.037 \\
\hline Social Function & $7.7(-3.5,18.9)$ & 0.145 & $9.4(4.5,13.2)$ & 0.011 & $11.4(-1.1,23.8)$ & 0.070 \\
\hline Role of Emotional & $1.1(-2.7,5.1)$ & 0.482 & $4.7(1.4,8.6)$ & 0.021 & $2.6(-10.2,15.5)$ & 0.675 \\
\hline Mental Health & $2.4(-6.8,13.5)$ & 0.564 & $6.4(2.4,10.4)$ & 0.004 & $5.3(-10.9,21.3)$ & 0.490 \\
\hline Vitality & $3.2(-0.7,7.3)$ & 0.093 & $10(1.6,18.2)$ & 0.216 & $6.8(-10,23.7)$ & 0.429 \\
\hline
\end{tabular}


In the same vein of this study, Alemdaroğlu et al. suggested mild, submaximal strengthening exercises as an appropriate choice for Duchene muscular dystrophy (DMD) patients (14). Lott et al. confirmed the same findings for DMD ambulatory patients after performing home-based isometric strengthening exercise program on knee muscles and detecting strength improvements in knee flexors and extensors (27). Bankolé et al. reported decreased fatigue perception and 6-minute walk test and increased maximum muscle contraction after combined aerobic and strengthening exercises among FSHD patients (28). Conversely, Lindeman $e t a l$. showed that maximum voluntary contraction was not significantly changed after the strengthening training in myotonic dystrophy patients (29). These controversies could be explained with different primary muscle strengths before intervention and different age-gender-related combinations of participants.

Hammarén et al., studying balance training on myotonic dystrophy type 1 patients, found decreased falling, improved activities-specific balance confidence (ABC) scale, unchanged TUG and knee extensors strength, and increased time for the 10-meter walk. Authors interpreted findings that self-assessed balance confidence is impressed by rehabilitation program but not functional tests(30). In another study on myotonic dystrophy patients, improved BBS, fast gait speed, and muscle strength were observed; however, they did not assess balance and muscle strength relationship (31). Considering mentioned studies, using different tests with different levels of sensitivity could be responsible for detected discrepancies.

We found BBS changes more prominent in the EMG-biofeedback group in comparison to CG. Previous studies suggested that auditory or visual feedback during biofeedback endorses unused or underused synaptic pathways that cause motor learning. Additionally, EMG as a source of feedback leads to down-training of a hyperactive muscle or up-training a weak muscle, thus improving muscular control (16). These two mechanisms could explain better results in the EMG-B group regarding balance-related findings independent of muscle strengths.

Although we found some changes in some subscales of quality of life in our study after the intervention, since many potential confounding factors were not assessed in our research, it is better not to interpret these findings.
Quality of life improvements are not related only to rehabilitation programs, and other factors such as family or society qualification, career or economic condition could influence their quality of life (32).

We faced some limitations. Despite acceptable sample size, patients dropped out during training sessions, and follow-ups were considerable (40\%). However, considering COVID-19 pandemic effects and MD patients' disabilities, this drop-out rate is not surprising. Second, we did not use any objective tests such as imaging or laboratory tests to evaluate muscle damage. However, none of the participants left the study due to pain or progressive weakness, or injury. Third, it was better to select one type of MD for this study because of different protein involvement. Using different functional tests with a wide range of sensitivity is one of the strengths of this study.

\section{CONCLUSIONS}

This study demonstrated the benefits of a supervised strengthening exercise program in MD patients. These benefits seem to be occurred without any adverse effects to cause patients to drop out of the study. Clinicians might be encouraged to use EMG-based biofeedback in rehabilitation programs of MD patients, which could be more influential due to motor control improvements, especially on their balance, leading to decrease fallings and its catastrophic consequences. Studies with a focused evaluation of one specific type of MDs with appropriate sample size and longer follow-up is recommended.

\section{FUNDINGS}

This study had a funding source of School of Medicine. The final article has been extracted from the thesis written by Dr. N.M., School of Medicine, Tehran University of Medical Sciences. It was also approved by the Physical Medicine and Rehabilitation Research Center of Shariati Educational Hospital.

\section{CONFLICT OF INTERESTS}

The authors declare that they have no conflict of interests. 


\section{REFERENCES}

1. Mercuri E, Muntoni F. Muscular dystrophies. Lancet 2013;381(9869):845-60.

2. Tesei A, Nobile M, Colombo P, et al. Mental health and coping strategies in families of children and young adults with muscular dystrophies. J Neurol 2020;267(7):2054-69.

3. Berends J, Tieleman AA, Horlings CG, et al. High incidence of falls in patients with myotonic dystrophy type 1 and 2: a prospective study. Neuromuscul Dis 2019;29(10):758-65.

4. Roussel M-P, Morin M, Gagnon C, Duchesne E. What is known about the effects of exercise or training to reduce skeletal muscle impairments of patients with myotonic dystrophy type 1? A scoping review. BMC musculoskeletal disorders 2019;20(1):1-14.

5. Mankodi A, Azzabou N, Bulea T, et al. Skeletal muscle water T2 as a biomarker of disease status and exercise effects in patients with Duchenne muscular dystrophy. Neuromuscul Dis 2017;27(8):705-14.

6. Garrood P, Hollingsworth KG, Eagle M, et al. MR imaging in Duchenne muscular dystrophy: quantification of T1-weighted signal, contrast uptake, and the effects of exercise. J Magn Reson Imaging: An Official Journal of the International Society for Magnetic Resonance in Medicine 2009;30(5):1130-8.

7. Tagliavini F, Sardone F, Squarzoni S, et al. Ultrastructural changes in muscle cells of patients with collagen VI-related myopathies. Muscles Ligaments Tendons J 2013;3(4):281.

8. Pisconti A, Bernet JD, Olwin BB. Syndecans in skeletal muscle development, regeneration and homeostasis. Muscles Ligaments Tendons J 2012;2(1):1.

9. Sveen ML, Jeppesen TD, Hauerslev S, Køber L, Krag TO, Vissing J. Endurance training improves fitness and strength in patients with Becker muscular dystrophy. Brain 2008;131(11):2824-31.

10. Kierkegaard M, Harms-Ringdahl K, Edström L, Holmqvist LW, Tollbäck A. Feasibility and effects of a physical exercise programme in adults with myotonic dystrophy type 1: a randomized controlled pilot study. J Rehabil Med 2011;43(8):695-702.

11. Jansen M, van Alfen N, Geurts AC, de Groot IJ. Assisted bicycle training delays functional deterioration in boys with Duchenne muscular dystrophy: the randomized controlled trial "no use is disuse”. Neurorehabil Neural Repair 2013;27(9):816-27.

12. Lindeman E, Leffers P, Spaans F, et al. Strength training in patients with myotonic dystrophy and hereditary motor and sensory neuropathy: a randomized clinical trial. Arch Phys Med Rehabil 1995;76(7):612-20.

13. Van der Kooi E, Vogels O, van Asseldonk R, et al. Strength training and albuterol in facioscapulohumeral muscular dystrophy. Neurology 2004;63(4):702-8.

14. Alemdaroğlu I, Karaduman A, Yilmaz ÖT, Topaloğlu H. Different types of upper extremity exercise training in Duchenne muscular dystrophy: effects on functional performance, strength, endurance, and ambulation. Muscle Nerve 2015;51(5):697-705.

15. Voet N, Bleijenberg G, Hendriks J, et al. Both aerobic exercise and cognitive-behavioral therapy reduce chronic fatigue in FSHD: an RCT. Neurology 2014;83(21):1914-22.

16. Huang H, Wolf SL, He J. Recent developments in biofeedback for neuromotor rehabilitation. J Neuroeng Rehabil 2006;3(1):1-12.
17. Basmajian JV. Research foundations of EMG biofeedback in rehabilitation. Biofeedback Self Regul 1988;13(4):275-98.

18. Padulo J, Oliva F, Frizziero A, Maffulli N. Basic principles and recommendations in clinical and field science research: 2018 update. Muscle Ligaments Tendons J 2018;8(3):305-7.

19. Beenakker E, Van der Hoeven J, Fock J, Maurits N. Reference values of maximum isometric muscle force obtained in 270 children aged 4-16 years by hand-held dynamometry. Neuromuscul Dis 2001;11(5):441-6.

20. De Lattre C, Payan C, Vuillerot C, et al. Motor function measure: validation of a short form for young children with neuromuscular diseases. Arch Phys Med Rehabil 2013;94(11):2218-26.

21. Krupp LB, LaRocca NG, Muir-Nash J, Steinberg AD. The fatigue severity scale: application to patients with multiple sclerosis and systemic lupus erythematosus. Arch Neurol 1989;46(10):1121-3.

22. Lue Y-J, Lin R-F, Chen S-S, Lu Y-M. Measurement of the functional status of patients with different types of muscular dystrophy. Kaohsiung J Med Sci 2009;25(6):325-33.

23. Podsiadlo D, Richardson S. The timed "Up \& Go": a test of basic functional mobility for frail elderly persons. J Am Geriatr Soc 1991;39(2):142-8.

24. Kubori Y, Matsuki R, Hotta A, Morisawa T, Tamaki A. Comparison between stair-climbing test and six-minute walk test after lung resection using video-assisted thoracoscopic surgery lobectomy. J Phys Ther Sci 2017;29(5):902-4.

25. Duncan MJ, Lawson C, Walker LJ, Stodden D, Eyre EL. The utility of the supine-to-stand test as a measure of functional motor competence in children aged 5-9 years. Sports 2017;5(3):67.

26. Franjoine MR, Gunther JS, Taylor MJ. Pediatric balance scale: a modified version of the berg balance scale for the school-age child with mild to moderate motor impairment. Pediatr Phys Ther 2003;15(2):114-28.

27. Lott DJ, Taivassalo T, Cooke KD, et al. Safety, feasibility, and efficacy of strengthening exercise in Duchenne muscular dystrophy. Muscle Nerve 2021;63(3):320-6.

28. Bankolé L-C, Millet GY, Temesi J, et al. Safety and efficacy of a 6-month home-based exercise program in patients with facioscapulohumeral muscular dystrophy: a randomized controlled trial. Medicine 2016;95(31):e4497.

29. Lindeman E, Spaans F, Reulen J, Leffers P, Drukker J. Progressive resistance training in neuromuscular patients. Effects on force and surface EMG. J Electromyogr Kinesiol 1999;9(6):379-84.

30. Hammarén E, Lindberg C, Kjellby-Wendt G. Effects of a balance exercise programme in myotonic dystrophy type 1 : a pilot study. Eur J Physiother 2015;17(3):123-31.

31. Missaoui B, Rakotovao E, Bendaya S, et al. Posture and gait abilities in patients with myotonic dystrophy (Steinert disease). Evaluation on the short-term of a rehabilitation program. Ann Phys Rehabil Med 2010;53(6-7):387-98.

32. Grootenhuis MA, De Boone J, Van der Kooi AJ. Living with muscular dystrophy: health related quality of life consequences for children and adults. Health Qual Life Outcomes 2007;5(1):1-8. 